\title{
COMPARISON OF VARIOUS DISTRIBUTONS USED FOR COLLAGEN FIBRE ORIENTATIONS IN ARTERIAL LAYERS
}

\author{
J. Fischer*, J. Burša**
}

\begin{abstract}
To consider tissue anisotropy in computational models of arterial wall, information on directions of collagen fibres and their dispersion in the individual layers of the wall is needed. Many papers introduce the assumption of two fibre families although its experimental substantiation is poor. In this paper we analyse data on direction distribution of collagen fibres published in several papers, in contrast to their authors, without any anticipatory assumptions. Histograms and their different mathematical representations are compared by means of coefficient of determination $R^{2}$ to obtain the best representation of the experimental data. The results show that in most of the analysed cases other distributions give better representation of the experimental data; specifically, the presented bimodal fit was surpassed with uni-, trior quadri-modal distributions or even with isotropic distribution with a large noise. This effect could be even enhanced when information criteria were applied. Finally, the best mathematical representations of the data (the best fits) are transformed into parameters used by anisotropic constitutive models in FEM analyses.
\end{abstract}

Keywords: collagen fibres, direction distribution, arterial tissue

\section{Introduction}

Arterial tissues are reinforced with collagen fibres causing their anisotropic mechanical properties. For their description many different constitutive models were developed (e.g. Lanir, 1983, Fung, 1993, Holzapfel et al., 2000, Holzapfel et al., 2005, Gasser et al., 2006). Among them structure based models can bring the best results if mechanical testing under different biaxial stresses is completed with histological information on directions of collagen fibres in the tissue including their dispersion (Polzer et al., 2015). Without structural information the advantages of these anisotropic models cannot be exploited, and its lack represents a severe limitation in stress-strain modelling of arterial and other soft tissues. Since the constitutive model with two perfectly aligned symmetric fibre families was published (Holzapfel et al., 2000), the existence two fibre families have been broadly accepted although it was not rigorously supported by experiments. The supervening substantiation of their existence in arterial tissue is not convincing; many authors intrinsically assume two collagen fibres in the tissue (Schrauwen, et al., 2012, Schriefel et al., 2011, Schriefel et al., 2012) and nobody has compared different fits to experimental data, i. e. to histograms of direction distribution of collagen fibres. To fill this gap, this paper focuses on analysis of some histograms published in literature and comparison of their different mathematical approximations; this issue is decisive for rigorous application of anisotropic hyperelastic constitutive models based on the number of fibre families, their main directions and dispersion around these directions. Although there are models taking also fibre waviness into consideration (Martuffi et al., 2011), this issue remains out of scope of this paper due to a lack of applicable experimental data.

\section{Mathematical methods}

The most frequently used model used to fit the histograms with a dominant direction and some dispersion is $\pi$-periodic von Mises distribution given by the formula (adapted from Schriefel et al., 2012):

* Bc. Jiří Fischer: Institute of Solid Mechanics, Mechatronics and Biomechanics, Brno University of Technology,Technická 2896/2; 61669 Brno;CZ,182495@vutbr.cz

** Prof. Ing. Jiř́ Burša, PhD.: Institute of Solid Mechanics, Mechatronics and Biomechanics, Brno University of Technology, Technická 2896/2; 61669 Brno; CZ, bursa@fme.vutbr.cz 


$$
\rho(\phi)=a \cdot \frac{\exp (b \cos (2(\phi-\mu)))}{2 \pi I_{0}(b)}
$$

where angle $\phi$ defines the direction of an individual fibre, $b$ is a concentration parameter describing the dispersion of fibre directions ( $b=\infty$ for isotropic distribution and $b=0$ for perfectly aligned fibres), $a$ stands as normalizing parameter, angle $\mu$ defines the mean fibre orientation in the family, and $I_{0}(b)$ is a modified Bessel function of the first kind of order 0. Eq. (1) represents a unimodal distribution, i.e. we assume just one fibre family. For more fibre families the most frequently applied model is given by combination of two (for $\mathrm{n}=2$ ) or more (for $\mathrm{n} \geq 3$ ) von Mises distributions:

$$
\rho(\phi)=a \cdot \sum_{i=1}^{n} \frac{\exp \left(b_{i} \cos \left(2\left(\phi-\mu_{i}\right)\right)\right)}{2 \pi I_{0}\left(b_{i}\right)} .
$$

In contrast, other authors (Schrauwen et al., 2012) use the following equation for unimodal distribution:

$$
\rho\left(\phi, \phi_{1}, \sigma\right)=A \exp \frac{\cos \left(2\left(\phi-\phi_{1}\right)\right)+1}{\sigma},
$$

where $\phi$ is the transmural angle, $\phi_{1}, \sigma$ are parameters of circular 3D distribution and parameter $A$ is a normalization factor. In most papers two fibre families in symmetric helical arrangement are assumed and thus a modified form of 3D distribution (3) is used, given by the following formula:

$$
\rho\left(\alpha, \alpha_{1}, \alpha_{2}, \sigma_{1}, \sigma_{2}\right)=A\left(\exp \frac{\cos \left(2\left(\alpha-\alpha_{1}\right)\right)+1}{\sigma_{1}}+\exp \frac{\cos \left(2\left(\alpha-\alpha_{2}\right)\right)+1}{\sigma_{2}}\right) .
$$

Angles $\alpha_{1}, \alpha_{2}$ are mean angles of both fibre families and $\sigma_{1}, \sigma_{2}$ correspond to their widths (dispersion). Also here $A$ represents a normalization factor. Unfortunately, there is no information on the way of fitting in (Schrauwen et al., 2012). Thus, it is not clear whether $A$ is the same for both parts of the bimodal distribution fitted simultaneously, or whether the $A$ values are different, and fitting was made separately for both fibre families.

The last mathematical model used for comparison was the von Mises distribution modified in order to meet the normalization condition as follows (Gasser et al., 2006):

$$
\frac{1}{4 \pi} \int_{\omega} \rho(M(\theta, \phi)) \mathrm{d} \omega=1,
$$

where $M$ is an arbitrary unit vector in the 3D Eulerian space, $\theta$ and $\phi$ are Eulerian angles, $\omega$ is a unit sphere and $\mathrm{d} \omega=\sin \theta \mathrm{d} \theta \mathrm{d} \phi$. Finally, the probability density function according to (5) is given by:

$$
\rho(\theta)=4 \sqrt{\frac{b}{2 \pi}} \frac{\exp (b(\cos (2 \theta)+1))}{\operatorname{erfi}(\sqrt{2 b})},
$$

where $\operatorname{erfi}(x)=-\mathrm{i} \operatorname{erf}(x)$ means the imaginary error function and $b$ has the same meaning of concentration parameter as in (1). Alternatively, directions in a fibre family can be specified using dispersion parameter $\kappa$ (see Fig. 1) which is given by the formula (Schriefel et al, 2011):

$$
\kappa=\frac{1}{4} \int_{0}^{\pi} \rho(\theta) \sin ^{3} \theta \mathrm{d} \theta
$$

where $\rho(\theta)$ is distribution from equation (6). Relation between both parameters can be seen in Fig. 1.

The distributions listed above are mutually compared using data presented in literature to decide which can give better representation of the specific collagen fibres distributions obtained experimentally.

\section{Results}

In this paper we compare the quality of fit of distributions published in two papers. In none of them raw data was published, thus conversion of the histograms into numbers was done using open source software. Specifically, the image with histogram was loaded into the PlotDigitizer program and the 
individual columns were manually clicked on their top to transform this position into numerical values of coordinates. Then the array with the obtained $\mathrm{x}$ and $\mathrm{y}$ coordinates was recorded for further processing.

In (Schrauwen et al., 2012) the data was fitted with a bimodal distribution without having specified the quality of the fits. We replicated their fits and compared their quality with a unimodal distribution by means of coefficient of determination $\mathrm{R}^{2}$; in our fitting procedure, equation (1) was used. In some cases, the unimodal distribution has shown a comparable or even better quality of the fit. In one case (for 40 $\mathrm{mmHg}$ ) neither bimodal nor unimodal distributions resulted in an acceptable fit; the best one (although still not good) was obtained with isotropic (constant) distribution of fibre directions (see Table1).

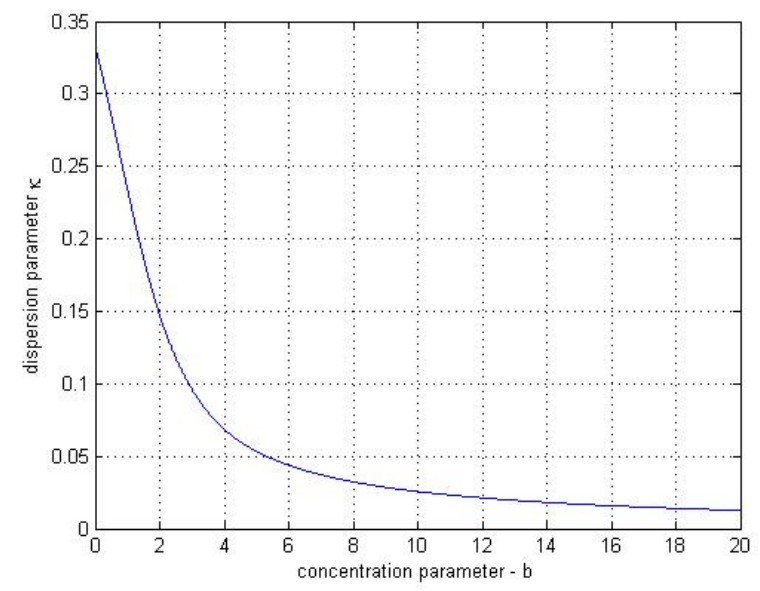

Figure 1: Relation between dispersion parameter $\kappa$ and concentration parameter $b$.

Table 1: Comparison of unimodal and bimodal fits to data from (Schrauwen et al., 2012) obtained under varying arterial pressure. Values $b, a$ and $\mu$ are parameters of unimodal von Mises distribution (eq. (1)).

\begin{tabular}{|c|c|c|l|l|l|}
\hline pressure $[\mathrm{mmHg}]$ & $\mathrm{R}^{2}$ bimodal eq. (3) & $\mathrm{R}^{2}$ unimodal eq. (1) & \multicolumn{1}{|c|}{$b$} & \multicolumn{1}{|c|}{$a$} & $\mu[\mathrm{rad}]$ \\
\hline 0 & 0.46 & 0.84 & 1.064 & 1.908 & -0.56 \\
\hline 40 & -0.17 & 0.00 (constant) & 0 & 1.99 & - \\
\hline 80 & 0.83 & 0.64 & 2.276 & 1.865 & 1.188 \\
\hline 120 & 0.83 & 0.80 & 3.267 & 1.983 & 1.384 \\
\hline
\end{tabular}

A similar comparison was done for the data from (Schriefel et al, 2011); the dispersion parameter $\kappa$ published there was recalculated into concentration parameter $b$ for easier comparison (see eqs. (6), (7) and figure 1). Although the authors state there are $2 \div 4$ fibre families in the intima of the investigated arteries, they still work with the bimodal distribution only. In contrast, we used multimodal distributions for intima, because histograms actually showed three (for CI) or four (for A or T) peaks. Specifically, eq. (2) was used in its extended form for trimodal or quadrimodal distributions. Thus, we were able to achieve a significantly better fit (with higher $\mathrm{R}^{2}$ ) of the histograms than in the original paper (see Table 2). On the other hand, unimodal distribution made here for comparison using eq. (1) gave us a significantly worse fit in comparison with multimodal or bimodal ones.

For media layers the analysis was also made using equations (1) and (2). For the common iliac artery unimodal distribution (with circumferential orientation of fibres) showed a better fit while a symmetric bimodal distribution was confirmed for both thoracic and abdominal aortas. Higher $\mathrm{R}^{2}$ values were achieved with our bimodal distribution because we have neglected the elevation angles of fibres (highly constrained by small thickness of the layer) and applied a 2D distribution only, in contrast to a 3D analysis used in (Schriefel et al., 2011). 
Table 2: Comparison of our fits to data from (Schriefel et al, 2011). Subscripts old and new refer to original published data and our new fits, respectively. T, A, and CI mean thoracic aorta, abdominal aorta, and common iliac artery, respectively.

\begin{tabular}{|c|c|c|c|c|c|}
\hline location & $b_{\text {old }}$ & $R_{\text {old }}^{2}$ & $R_{\text {new }}^{2}$ & $b_{\text {new }}$ & $\mu[\mathrm{rad}]$ \\
\hline $\mathbf{C I}$ - intima & 4.8885 & 0.18 & 0.44 & $2.212,2.451,2.448$ & $-1.5,0.618,-0.606$ \\
\hline $\mathbf{A}$ - intima & 5.532 & 0.55 & 0.79 & $1.266,2.291,2.228,1.694$ & $0.049,-0.539,0.580,1.500$ \\
\hline $\mathbf{T}-$ intima & 5.1425 & 0.60 & 0.72 & $2.230,2.066,1.142,1.851$ & $0.784,-0.772,-1.420,-0.100$ \\
\hline $\mathbf{C I}$ - intima & 4.8885 & 0.18 & 0.03 & 0.0884 & -0.6983 (unimodal) \\
\hline $\mathbf{A}$ - intima & 5.532 & 0.55 & 0.45 & 0.3658 & 0.02724 (unimodal) \\
\hline $\mathbf{T}-$ intima & 5.1425 & 0.60 & 0.19 & 0.1872 & 0.0374 (unimodal) \\
\hline $\mathbf{C I}-$ media & 4.5225 & 0.63 & 0.95 & 2.302 & -0.016 (unimodal) \\
\hline $\mathbf{A}-$ media & 6.7147 & 0.82 & 0.96 & 3.930 & \pm 0.408 (bimodal) \\
\hline $\mathbf{T}-$ media & 5.7546 & 0.74 & 0.96 & 3.477 & \pm 0.474 (bimodal) \\
\hline
\end{tabular}

\section{Conclusion}

Mathematical comparison of quality of the fit for different distribution functions has shown that some literature data on distribution of collagen fibres in arterial layers do not in fact correspond to bimodal distribution assumed by many authors and can be fitted much better with others, often unimodal distributions. This tendency might become even more pronounced if information criteria were used. For instance, Akaike information criterion (AIC) is going to be applied in our future work; as an estimator of the relative quality of statistical models it prevents the risk of overfitting or underfitting. Consequently, no anticipatory assumptions and much more sophisticated analyses of experimental data on fibre directions should be used to transform them into structural parameters of constitutive models capable to achieve a credible description of mechanical properties of arterial layers.

\section{Acknowledgement}

This work was supported by Czech Science Foundation, project No. 18-13663S.

\section{References}

Fung Y.C. (1993) Biomechanics. Mechanical properties of living tissues. Springer New York Berlin Heidelberg

Gasser T.C., Ogden R.W and Holzapfel G.A. (2006) Hyperelastic modelling of arterial layers with distributed collagen fibre orientation. Journal of The Royal Society Interface, 3, 15-35

Holzapfel G.A. Gasser T.C., and Ogden R.W (2000) A New Constitutive Framework for Arterial Wall Mechanics and a Comparative Study of Material Models. Journal of Elasticity, 61, 1573-2681

Holzapfel G.A. (2005) Hyperelastic modelling of arterial layers with distributed collagen fibre orientation, Journal of The Royal Society Interface, 3, 15-35

Lanir Y. (1983) Constitutive equations for fibrous connective tissue. Journal of Biomechanics, 16, 1-12

Martufi G. and Gasser T.C. (2011) A constitutive model for vascular tissue that integrates fibril, fiber and continuum levels with application to the isotropic and passive properties of the aorta. J Biom, 44, 2544-2550

Plot Digitizer [open source software]. (Version 2.6.8; 2015). Retrieved from http://plotdigitizer.sourceforge.net/

Polzer S., Gasser T.C., Novak K., Man V., Tichy M., Skacel P., Bursa J. (2015) Structure-based constitutive model can accurately predict planar biaxial properties of aortic wall tissue. Acta Biomaterialia, 14, 133-145

Schrauwen J.T.C, Vilanova A., Rezakhaniha R., Stergiopulos N., van de Vosse F.N. and Bovendeerd, P.H.M. (2012) A method for the quantification of the pressure dependent 3D collagen configuration in the arterial adventitia. Journal of Structural Biology, 180, 335-342

Schriefel A.J., Zeindlinger G., Pierce D.M., Regitnig P. and Holzapfel G.A. (2011) Determination of the layer-specific distributed collagen fibre orientations in human thoracic and abdominal aortas and common iliac arteries. Journal of The Royal Society Interface, 9, 1275-1286

Schriefel A.J., Reinisch A.J., Sankaran S., Pierce D.M., Holzapfel G. A. (2012) Quantitative assessment of collagen fibre orientations from two-dimensional images of soft biological tissues. J Royal Soc Interface, 9, 3081-3093 